\section{Josephson tunnel junctions with ferromagnetic $\mathrm{Fe}_{0.75} \mathrm{Co}_{0.25}$ barriers}

To cite this article: D Sprungmann et al 2009 J. Phys. D: Appl. Phys. 42075005

View the article online for updates and enhancements.

\section{Related content}

Spin-polarized supercurrents for spintronics: a review of current progress Matthias Eschrig

Spin-triplet supercurrent in Co-based Josephson junctions Mazin A Khasawneh, Trupti S Khaire Carolin Klose et al.

The effect of normal and insulating lavers on 0- transitions in Josephson junctions with a ferromagnetic barrier

D M Heim, N G Pugach, M Yu Kupriyanov et al.

\section{Recent citations}

- Edge currents in frustrated Josephson
Aunction ladders
A M Marques et al
- Spin-polarized supercurrents for
$\frac{\text { spintronics: a review of current progress }}{\text { Matthias Eschrig }}$
- Giant thermoelectric effects in a proximity-
$\frac{\text { coupled superconductor-ferromagnet }}{\text { device }}$
P Machon et al




\title{
Josephson tunnel junctions with ferromagnetic $\mathrm{Fe}_{0.75} \mathrm{Co}_{0.25}$ barriers
}

\author{
D Sprungmann ${ }^{1}$, K Westerholt ${ }^{1}, \mathrm{H} \mathrm{Zabel}^{1}, \mathrm{M}$ Weides $^{2}$ and H Kohlstedt $^{2}$ \\ ${ }^{1}$ Institut für Experimentalphysik/Festkörperphysik, Ruhr-Universität Bochum, 44780 Bochum, Germany \\ ${ }^{2}$ Institute of Solid State Research and JARA-Fundamentals of Future Information Technology, Research \\ Centre, Jülich, 52425 Jülich, Germany
}

Received 10 December 2008, in final form 19 February 2009

Published 20 March 2009

Online at stacks.iop.org/JPhysD/42/075005

\begin{abstract}
Josephson tunnel junctions with the strong ferromagnetic alloy $\mathrm{Fe}_{0.75} \mathrm{Co}_{0.25}$ as the barrier material were studied. The junctions were prepared with high quality down to a thickness range of a few monolayers of $\mathrm{Fe}-\mathrm{Co}$. An oscillation length of $\xi_{\mathrm{F} 2} \approx 0.79 \mathrm{~nm}$ between 0 and $\pi$-Josephson phase coupling and a very short decay length $\xi_{\mathrm{F} 1} \approx 0.22 \mathrm{~nm}$ for the amplitude of the superconducting pair wave function in the $\mathrm{Fe}-\mathrm{Co}$ layer were determined. The rapid damping of the pair wave function inside the $\mathrm{Fe}-\mathrm{Co}$ layer is caused by the strong ferromagnetic exchange field and additional magnetic pair breaking scattering. Josephson junctions with $\mathrm{Fe}-\mathrm{Co}$ barriers show a significantly increased tendency towards magnetic remanence and flux trapping for larger thicknesses $d_{\mathrm{F}}$.
\end{abstract}

(Some figures in this article are in colour only in the electronic version)

\section{Introduction}

The proximity effect in thin film heterostructures combining superconducting $(\mathrm{S})$ and ferromagnetic $(\mathrm{F})$ layers is a topic of great current interest [1]. For example, it was shown that the critical temperature $T_{\mathrm{c}}$ depends on the thickness and magnetic orientation of F layers in SF bi- or multilayers [2-4]. Another closely related phenomenon is the occurrence of so-called $\pi$-coupling in SFS-type Josephson junctions (JJs). For certain thickness ranges of the ferromagnetic layer $d_{\mathrm{F}}$ the Josephson coupling energy $E_{\mathrm{J}}$ has a minimum for a phase difference $\varphi=\pi$ and not for $\varphi=0$ as in normal Josephson junctions. The ground state of the Josephson junction oscillates between 0 and $\pi$ coupling with the oscillation period given by $\Delta d_{\mathrm{F}}=\pi \xi_{\mathrm{F}}$. The magnitude of the magnetic length $\xi_{\mathrm{F}}$ depends on the kind of transport regime. In the clean limit, i.e. when for the electron mean free path $\ell_{\mathrm{m}}$ in the ferromagnet $\ell_{\mathrm{m}} \gg \xi_{\mathrm{F}}$ holds, it is determined by $\xi_{\mathrm{F}}=\hbar v_{\mathrm{F}} / E_{\mathrm{ex}}$, with the Fermi velocity $v_{\mathrm{F}}$, the exchange energy $E_{\text {ex }}$ and Planck's constant $\hbar$. In the dirty limit, i.e. if $\ell_{\mathrm{m}} \ll \xi_{\mathrm{F}}, \xi_{\mathrm{F}}$ depends on the electron diffusion constant $D_{\mathrm{F}}=\frac{1}{3} v_{\mathrm{F}} \ell_{\mathrm{m}}$ via

$$
\xi_{\mathrm{F}}=\sqrt{\frac{\hbar D_{\mathrm{F}}}{E_{\mathrm{ex}}}}
$$

For SIFS-type junctions in the dirty limit with magnetic scattering the critical current is given by $[5,6]$

$I_{\mathrm{c}} R_{\mathrm{n}}\left(d_{\mathrm{F}}\right) \propto\left|\cos \left(\frac{d_{\mathrm{F}}-d_{0}}{\xi_{\mathrm{F} 2}}\right)\right| \exp \left(-\frac{d_{\mathrm{F}}}{\xi_{\mathrm{F} 1}}\right)$,

where $d_{0}$ denotes the thickness of the non-magnetic (dead magnetic) part of the F-layer and I stands for the $\mathrm{AlO}_{x}$ tunnel barrier within the stack, $R_{\mathrm{n}}$ is the resistance in the normal state. In general the decay length $\xi_{\mathrm{F} 1}$ and the oscillation length $\xi_{\mathrm{F} 2}$ are different. In the limit $E_{\mathrm{ex}} \gg k_{\mathrm{B}} T_{\mathrm{c}}$ they are related by the equation [6]:

$$
\xi_{\mathrm{F} 1 \mathrm{~F} 2}=\xi_{\mathrm{F}} \sqrt{\frac{1}{\sqrt{1+\left(\frac{\hbar / \tau_{\mathrm{ic}}}{E_{\mathrm{ex}}}\right)^{2} \pm\left(\frac{\hbar / \tau_{\mathrm{ic}}}{E_{\mathrm{ex}}}\right)}}},
$$

where the positive and negative signs in the denominator refers to $\xi_{\mathrm{F} 1}$ and $\xi_{\mathrm{F} 2}$, respectively.

Only if the pair breaking scattering rate $\tau_{\mathrm{ie}}^{-1}$ is small, i.e. $\hbar / \tau_{\mathrm{ie}} \ll E_{\mathrm{ex}}, \xi_{\mathrm{F} 1} \approx \xi_{\mathrm{F} 2}$ holds. For strong pair breaking scattering they differ and $\xi_{\mathrm{F} 1}<\xi_{\mathrm{F} 2}$.

The experimental confirmation of $\pi$ coupling in the SFS-type [7-9] as well as in the SIFS-type JJs [5, 10], promoted an upsurge of interest in JJs with ferromagnetic barriers. This is motivated by the perspective of applying $\pi$-coupled Josephson junctions in flux qubits or digital logic circuits [11]. For 
example, by combining a tunnel 0 - and a metallic $\pi-\mathrm{JJ}$ into one superconducting loop, one can define a quantum mechanical superconducting two level system with all properties required for the basic unit of quantum computational devices, the so-called qubits [12]. The physics of fractional vortices can also be studied in junctions with combined 0 and $\pi$ coupling [13].

For the superconducting layer $\mathrm{S}$ the elemental superconductor $\mathrm{Nb}$ is nearly exclusively used in the literature $[5,8,10$, 14-16]. For the F-layer either diluted ferromagnets such as PdNi [10], $\mathrm{NiCu}[5,14]$ or $\mathrm{SiFe}$ [15] or elemental 3d magnets are applied. In diluted ferromagnetic alloys the pair breaking of the Cooper pairs by the exchange field is weak and the oscillation length can be rather large, thus enabling for large thicknesses $d_{\mathrm{F}}$ a still high superconducting critical current density $j_{\mathrm{c}}$. This simplifies the observation of oscillations in $I_{\mathrm{c}}\left(d_{\mathrm{F}}\right)$, which is taken as the main experimental evidence for $\pi$ coupling. The first minimum ( 0 to $\pi)[5,10]$ and also the second minimum ( $\pi$ to 0 ) [14] was observed in JJs using $\mathrm{Ni}-\mathrm{Cu}$ alloys.

Junctions with $3 \mathrm{~d}$ transition metals such as $\mathrm{Fe}, \mathrm{Co}$ and $\mathrm{Ni}$ as the barrier material were studied in $[9,16,17]$. By using strong ferromagnets as the barrier material one expects a very short oscillation period, corresponding to a thickness of a few monolayers only. From the experimental point of view it is challenging to grow homogeneous barriers to obtain junctions with a sufficient comparability, which is crucial to observe the 0 to $\pi$ transition. Small lateral inhomogeneities in the ferromagnetic layer would severely deteriorate the junction properties by locally inducing phase shifts in the oscillating pair density. The competition between the short oscillation period and the exponential damping of the critical current density caused by the strong pair breaking from the exchange field characterizes the shape of the $I_{\mathrm{c}}\left(d_{\mathrm{F}}\right)$-dependence. Since the ratio $\xi_{\mathrm{F} 1} / \xi_{\mathrm{F} 2}$ determines the location of the $0-\pi$ transition, one can estimate the crossover thickness $d_{\mathrm{F}}^{0-\pi}$ even if the crossover is not observable directly. The alloy $\mathrm{Fe}_{0.75} \mathrm{Co}_{0.25}$, which we chose for the F-layer, has the largest magnetic moment of about $2.5 \mu_{\mathrm{B}}$ per atom in the bulk among the $3 \mathrm{~d}$ transition metal series. Therefore, we expect a large exchange energy and correspondingly very short coherence lengths which should be smaller than those of comparable $\mathrm{Fe}$ or $\mathrm{Ni}$ junctions. The value of the exchange energy of $\mathrm{Fe}_{0.75} \mathrm{Co}_{0.25}$ must be considered as the maximum value possible for SFS/SIFS junctions, based on 3d transition metals. Until now the composition $\mathrm{Fe}_{0.75} \mathrm{Co}_{0.25}$ has not been studied in the context of $\pi$ junctions.

\section{Preparation and experimental}

The junctions were fabricated using a combination of dc-magnetron sputtering and optical lithography, as described in detail in [18]. The schematic design of the junctions is depicted in figure 1 . The complete thin film stack including the barrier and a part of the top $\mathrm{Nb}$ electrode was sputtered in one run in a commercial sputtering chamber (Leybold Univex $450 \mathrm{~B}$ ) with a base pressure of $4 \times 10^{-7}$ mbar on a thermally oxidized Si substrate at room temperature, see figure 1 .

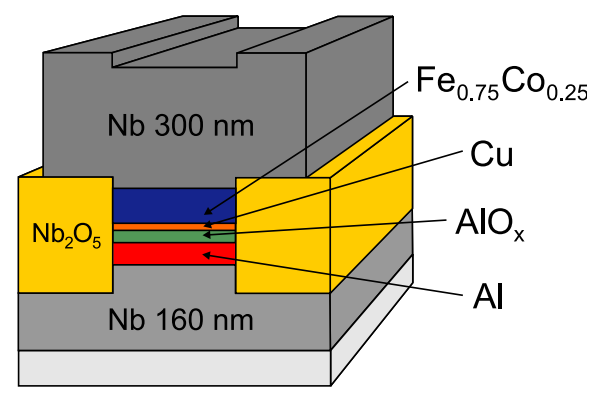

Figure 1. (Colour online.) Schematic design of the SINFS-type junctions, the insulation is prepared by using an anodic oxidation technique.

After sputtering a $(\mathrm{Nb}(40 \mathrm{~nm}) \mathrm{Al}(2.4 \mathrm{~nm}))_{4}$ multilayer, the $5 \mathrm{~nm}$ thick Al layer was deposited and thermally oxidized for $30 \mathrm{~min}$ in pure oxygen at pressures of $1.5 \mathrm{mbar}$ and $0.015 \mathrm{mbar}$, respectively. By using an oxidation pressure of 0.015 mbar instead of 1.5 mbar the $\mathrm{AlO}_{x}$ layer is definitely thinner and the critical current density increases by a factor of 12-15. This is helpful to improve the signal quality for larger $\mathrm{Fe}-\mathrm{Co}$ thicknesses, for which we expected a significant suppression of the supercurrent. The purpose of the thin Al interlayers in the $\mathrm{Nb} / \mathrm{Al}$ multilayer is the optimization of the top $\mathrm{Nb} / \mathrm{Al}$ interface in order to reduce the barrier roughness to a minimum. Depending on the oxygen pressure, about 1-2 nm of the $\mathrm{Al}$ layer are transformed into $\mathrm{AlO}_{x}$. The remaining metallic $\mathrm{Al}$ film becomes superconducting by the proximity effect below the transition temperature of $\mathrm{Nb}$. On top of the $\mathrm{AlO}_{x}$ layer a $2 \mathrm{~nm}$ thick non-magnetic (N) Cu film is sputtered, followed by the $\mathrm{Fe}-\mathrm{Co}$ ferromagnetic film. In recent work [18] it was shown that the $\mathrm{Cu}$ interlayer is needed to keep the interface roughness of the F layer on an acceptable level. The Fe-Co film is wedge shaped along the substrate length using the natural gradient of the sputtering rate. As a final step the $\mathrm{Nb}$-counter electrode with a thickness of $40 \mathrm{~nm}$ was deposited.

After the lift-off process mesas of $10 \times 50 \mu \mathrm{m}^{2}$ and $10 \times 200 \mu \mathrm{m}^{2}$ are defined by optical lithography and ion-beam etching. Afterwards the current leads and the sides of the stack are isolated by anodic oxidation of $\mathrm{Nb}$ [19]. Finally, after a short Ar plasma etching, the top electrode is completed by sputtering another $400 \mathrm{~nm}$ of $\mathrm{Nb}$.

For this study we prepared two series of junctions with two different $\mathrm{AlO}_{x}$ barrier thicknesses. The first series with the thinner $\mathrm{AlO}_{x}$ barrier covered the thickness range $d_{\mathrm{F}}=0.9$ $3.2 \mathrm{~nm}$ for the $\mathrm{Fe}-\mathrm{Co}$ layer and had a normal state resistance for the $10 \times 50 \mu \mathrm{m}^{2} \mathrm{JJs}$ of about $R_{\mathrm{n}}=0.16 \Omega$ and $R_{\mathrm{n}} \approx 0.052 \Omega$ $\left(10 \times 200 \mu \mathrm{m}^{2}\right)$, respectively. The second series with a thicker $\mathrm{AlO}_{x}$ barrier covered the thickness range $d_{\mathrm{F}}=0.4-1.1 \mathrm{~nm}$ and had $R_{\mathrm{n}} \approx 1.07 \Omega\left(10 \times 50 \mu \mathrm{m}^{2}\right)$.

The ferromagnetic properties of the thin Fe-Co layers were studied by a commercial SQUID magnetometer on identical, non-microstructured layer stacks covering the same Fe-Co thickness range. The $I-V$ characteristics of the Josephson junctions with and without an applied magnetic field in the film plane were measured at $T=4.2 \mathrm{~K}$ in a shielded ${ }^{4} \mathrm{He}$ cryostat using home made electronics. 


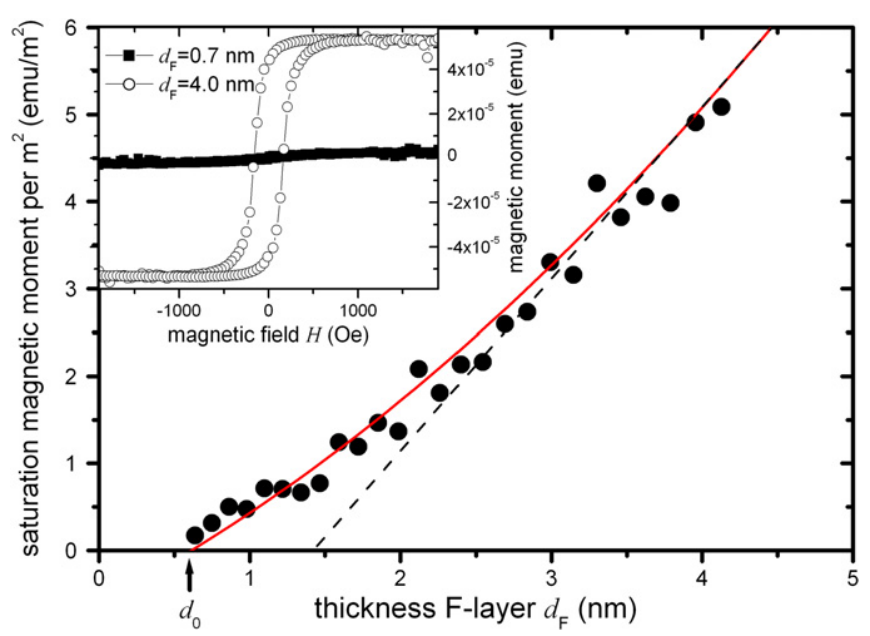

Figure 2. Saturation magnetic moment per $\mathrm{m}^{2}$ versus $\mathrm{Fe}-\mathrm{Co}$ thickness $d_{\mathrm{F}}$ measured at $T=15 \mathrm{~K}$. The dashed line is a theoretical curve corresponding to a magnetic moment of $2.5 \mu_{\mathrm{B}}$ /atom. The solid line leads to a total thickness of non-magnetic interlayers of $d_{0}=0.6 \mathrm{~nm}$. The inset depicts the hysteresis curves of two samples with different $\mathrm{Fe}-\mathrm{Co}$ layer thicknesses $d_{\mathrm{F}}=0.7 \mathrm{~nm}$ and $d_{\mathrm{F}}=4.0 \mathrm{~nm}$.

\section{Results and discussion}

The magnetism of the $\mathrm{Fe}_{0.75} \mathrm{Co}_{0.25}$ alloy layer in the JJs plays an important role, thus we first characterize the magnetic properties. The magnetic hysteresis loops of the SINFS stacks with $d_{\mathrm{F}}$ in the same range as for the JJs have been measured at $15 \mathrm{~K}$. In the inset of figure 2 we show two examples for a thickness $d_{\mathrm{F}}=0.7 \mathrm{~nm}$ and $d_{\mathrm{F}}=4.0 \mathrm{~nm}$. For the thicker film one observes a nearly square-shaped hysteresis loop with a coercive field of $200 \mathrm{Oe}$ and a saturation magnetization corresponding to about $70 \%$ of the bulk value. For the thinner film there is only a very weak ferromagnetic signal, indicating that due to intermixing at the interfaces very thin $\mathrm{Fe}-\mathrm{Co}$ layers become non-magnetic. The saturation magnetic moment normalized to the sample area is plotted versus the nominal thickness of the $\mathrm{Fe}-\mathrm{Co}$ layer in figure 2. Below a nominal thickness of $d_{\mathrm{F}}=0.6 \mathrm{~nm}$ the layers are nonferromagnetic. Above $d_{\mathrm{F}}=0.6 \mathrm{~nm}$ the ferromagnetic moment gradually increases and approaches the bulk moment of 2.5 $\mu_{B} /$ atom above about $d_{\mathrm{F}}=2.5 \mathrm{~nm}$. This indicates that at each interface by alloying with $\mathrm{Nb}$ or $\mathrm{Cu}$ there is a reduction in the ferromagnetic moment and the first two monolayers at each side are non-ferromagnetic.

A second experimental ingredient needed for a theoretical description of the Josephson junctions is the conduction electron mean free path of the ferromagnetic layer $\ell_{\mathrm{m}}$, which defines whether the dirty limit or the clean limit theory for the JJs applies. We measured the electrical conductivity of a single $\mathrm{Fe}_{0.75} \mathrm{Co}_{0.25}$ thin film of $8 \mathrm{~nm}$ thickness on thermally oxidized Si. The $\mathrm{Fe}_{0.75} \mathrm{Co}_{0.25}$-layer was capped with $30 \mathrm{~nm}$ of sputtered $\mathrm{SiO}_{2}$. The film had a very small residual resistivity ratio $\mathrm{RRR} \approx 1.2$ (defined as the ratio of the resistance measured at room temperature and at $4.2 \mathrm{~K}$ ) and a large residual resistivity of $\rho_{\mathrm{m}}=27 \mu \Omega \mathrm{cm}$. For Fe-Co in [21] a smaller value of $\rho_{\mathrm{m}}=14.8 \mu \Omega \mathrm{cm}$ was determined, indicating the

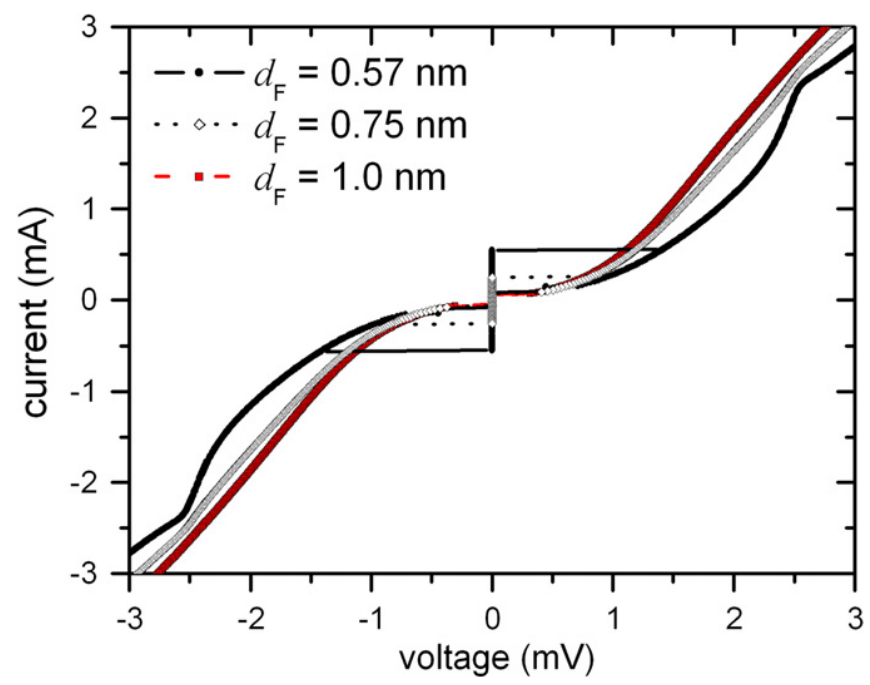

Figure 3. (Colour online.) $I-V$ curves of three junctions with different $\mathrm{Fe}-\mathrm{Co}$ thicknesses measured at $T=4.2 \mathrm{~K}$. The area of the junctions was $10 \times 50 \mu \mathrm{m}^{2}$.

sensitive influence of different growth conditions. The electron mean free path can be estimated using the standard free electron model formula [20]:

$$
\ell_{\mathrm{m}}=\frac{\pi^{2} k_{\mathrm{B}}^{2}}{e^{2} \rho_{\mathrm{m}} \gamma_{\mathrm{s}} v_{\mathrm{F}}}
$$

with the electronic specific heat coefficient $\gamma$ and the Fermi velocity $v_{\mathrm{F}}$. With $\gamma \approx 750 \mathrm{~J} \mathrm{~m}^{-3} \mathrm{~K}^{-2}$ and $v_{\mathrm{F}} \approx 1.56 \times$ $10^{6} \mathrm{~m} \mathrm{~s}^{-1}\left(\mathrm{Co}: v_{\mathrm{F}}=0.28 \times 10^{6} \mathrm{~m} \mathrm{~s}^{-1}\right.$, Fe: $v_{\mathrm{F}}=1.98 \times$ $10^{6} \mathrm{~m} \mathrm{~s}^{-1}$ [16]) we estimated $\ell_{\mathrm{m}}=0.23 \mathrm{~nm}$. This very small value for the mean free path indicates strong disorder scattering from the random distribution of $\mathrm{Fe}$ and $\mathrm{Co}$ atoms in the alloy. The value for $\ell_{\mathrm{m}}$ derived here must be considered as a rough estimate, because the growth on $\mathrm{SiO}_{2}$ is not directly comparable to the growth within the SINFS-stack.

In figure 3 we show the $I-V$ curves of junctions with different thicknesses of the ferromagnetic layer $d_{\mathrm{F}}$. One finds the typical features of Josephson tunnel junctions with ferromagnetic barriers $[5,10,18]$. For small thicknesses $d_{\mathrm{F}}$ the Josephson phase is strongly underdamped and the $I-V$ curves exhibit a pronounced hysteresis. For the lowest thickness in figure 3 one can resolve the double superconducting gap $2 \Delta$ of $\mathrm{Nb}$ at $2.7 \mathrm{meV}$. With increasing thickness $d_{\mathrm{F}}$, this feature and the hysteresis gradually vanish.

The Fraunhofer patterns $I_{\mathrm{c}}(H)$ of the same junctions are shown in figure $4(a)$. For $d_{\mathrm{F}}<1.1 \mathrm{~nm}$ we observe highly symmetric, periodic patterns with vanishing critical current at the minima, which is a clear indication of the homogeneity of both the $\mathrm{AlO}_{x}$ and $\mathrm{Fe}-\mathrm{Co}$ barriers in our samples. For thicknesses $d_{\mathrm{F}}>1.1 \mathrm{~nm}$ (see figure 4(b)) the global maximum of the Fraunhofer patterns was found to be increasingly shifted towards external fields $H \neq 0$, indicating the existence of an intrinsic magnetic stray field. Similar observations in Ni-based JJs have been published recently in [23]. In the case of $\mathrm{Ni}$ the flux trapping effects appear for thicknesses $d_{\mathrm{F}} \geqslant 3.7 \mathrm{~nm}$, which is significantly larger than in our samples. In the case of $\mathrm{Fe}_{0.75} \mathrm{Co}_{0.25}$ the magnetic stray 


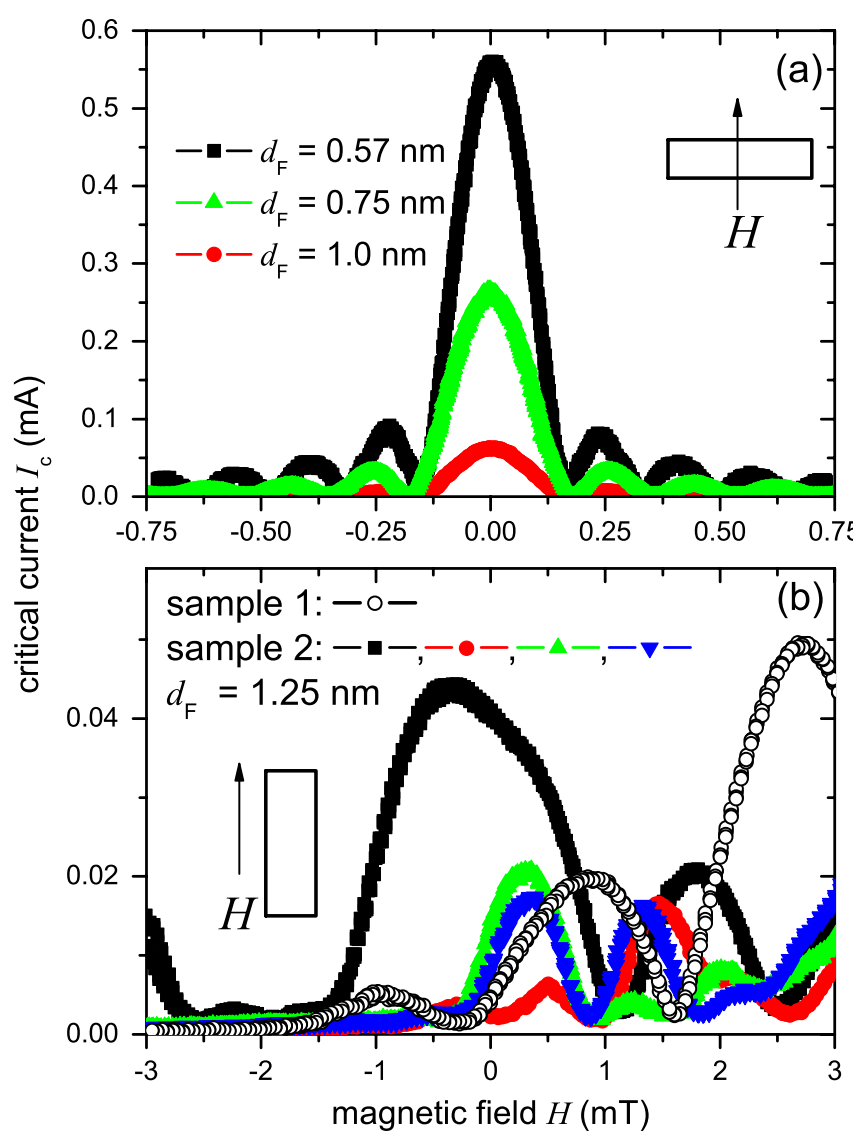

Figure 4. (Colour online.) (a) $H \perp$ long axis: in the thickness range $d_{\mathrm{F}}<1.1 \mathrm{~nm}$ the Fraunhofer patterns exhibit mirror symmetry. (b) $H \|$ long axis: for other junctions with thicknesses $d_{\mathrm{F}} \geqslant 1.1 \mathrm{~nm}$ flux trapping effects become inevitable. The solid symbols belong to one junction measured for various cooling cycles $(\Delta T \approx 200 \mathrm{~K})$. The junctions were cooled without applying an external magnetic field. The open circles belong to a sample with a similar thickness $d_{\mathrm{F}}$ showing the shift of the Fraunhofer pattern exemplarily. In both cases $(a)$ and $(b)$ the sample size was $10 \times 50 \mu \mathrm{m}^{2}$.

field appears within the 0 state, whereas in the case of $\mathrm{Ni}$ the samples are already in the $\pi$ state. The $I_{\mathrm{c}}(H)$ curve of one sample (open circles) in figure 4(b) appears to be shifted by $\sim 2.5 \mathrm{mT}$ along the field axis, corresponding to $2-3 \Phi_{0}$. In this case the external field was parallel to the long axis of the junction. If $H$ was orientated perpendicular to the long axis only extremely small critical currents could be measured even for maximum fields around $3 \mathrm{mT}$. This indicates the presence of a magnetic shape anisotropy within the F layer, which shifts the Fraunhofer pattern opposite to the magnetization direction [23]. Additionally, we usually found indication of some trapped magnetic flux, probably emanating from the ferromagnetic film and frozen in below $T_{\mathrm{c}}$. This flux leads to irregular, not reproducible $I_{\mathrm{c}}(H)$ patterns and the global maximum critical current $I_{\mathrm{c}}$ cannot be defined precisely. The maximum experimental critical current which is seen in the experiment is always an underestimation for the true maximum critical current. Additionally, this random error causes an increasing scattering of the data points for $d_{\mathrm{F}} \geqslant 1.1 \mathrm{~nm}$ in $I_{\mathrm{c}}\left(d_{\mathrm{F}}\right)$.

In figure 5 the global maximum critical current $I_{\mathrm{c}}$ determined by $I_{\mathrm{c}}(H)$ versus the nominal thickness of the

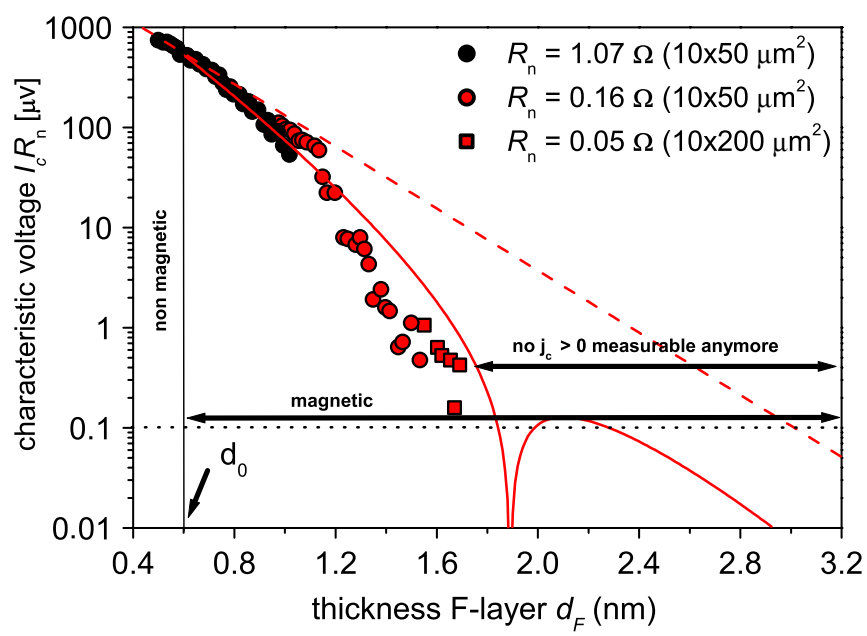

Figure 5. (Colour online.) $I_{\mathrm{c}} R_{\mathrm{n}}$ product versus $\mathrm{Fe}_{0.75} \mathrm{Co}_{0.25}$ thickness $d_{\mathrm{F}}$. The solid line is a theoretical curve corresponding to equation (1), the dashed curve corresponds to equation (2) for thicknesses $d_{\mathrm{F}} \leqslant 0.9 \mathrm{~nm}$. The dotted line marks the resolution limit of our instrumental setup. The parameters for the theoretical curve are $E_{\mathrm{ex}}=453 \mathrm{meV}, \xi_{\mathrm{F} 1}=0.22 \mathrm{~nm}, \xi_{\mathrm{F} 2}=0.79 \mathrm{~nm}, \xi_{\mathrm{F}}=0.42 \mathrm{~nm}$, $\ell_{\mathrm{m}}=0.23 \mathrm{~nm}, \hbar / \tau_{\mathrm{ie}}=1.66 E_{\mathrm{ex}}$. In order to compare both series with different $\mathrm{AlO}_{x}$ thicknesses the characteristic voltage $I_{\mathrm{c}} R_{\mathrm{n}}$ is plotted against $d_{\mathrm{F}}$. The squares and the circles are data points from samples which were produced within the same run. For thicknesses $d_{\mathrm{F}} \geqslant 1.7 \mathrm{~nm}$ no critical currents could be observed anymore.

ferromagnetic layers $d_{\mathrm{F}}$ is depicted. In fact, the product of the critical current and the normal state junction resistance $I_{\mathrm{c}} R_{\mathrm{n}}$ is plotted for a better comparison of the two series of samples with different thicknesses of the $\mathrm{AlO}_{x}$ barrier.

$I_{\mathrm{c}}$ is strongly damped for increasing $d_{\mathrm{F}}$ and reaches our experimental resolution limit of $I_{\mathrm{c}} R_{\mathrm{n}} \approx 0.1 \mu \mathrm{V}$ beyond $d_{\mathrm{F}}>$ $1.7 \mathrm{~nm}$.

For a quantitative analysis of $I_{\mathrm{c}} R_{\mathrm{n}}\left(d_{\mathrm{F}}\right)$ we divided the data into two sections, as done in the case of Ni-based SIFS junctions in [17]. For $d_{\mathrm{F}} \leqslant 0.6 \mathrm{~nm}$ the JJS are approximately treated as a SINS-type junction, with $E_{\mathrm{ex}}=0 \mathrm{meV}$. Then equation (1) converts into

$$
I_{\mathrm{c}} R_{\mathrm{n}}\left(d_{\mathrm{F}}\right) \propto \exp \left(-\frac{d_{\mathrm{F}}}{\xi_{\mathrm{N}}}\right)
$$

with the damping length

$$
\xi_{\mathrm{N}}=\sqrt{\frac{\hbar D_{\mathrm{N}}}{2\left(\pi k_{\mathrm{B}} T+\frac{\hbar}{\tau_{\mathrm{ie}}}\right)}}
$$

and the diffusion constant in the non-ferromagnetic layer $D_{\mathrm{N}}$ (see [6]). From the tangent at very thin Fe-Co layers $\left(d_{\mathrm{F}} \leqslant 0.6 \mathrm{~nm}\right)$ (see figure 5 , dashed line) we derive a very short damping length $\xi_{\mathrm{N}} \approx 0.28 \mathrm{~nm}$ and $I_{\mathrm{c}} R_{\mathrm{n}}\left(d_{\mathrm{F}}=\right.$ $0.6 \mathrm{~nm}) \approx 1 \mathrm{mV}$, corresponding to a magnetic scattering energy of $\hbar / \tau_{\mathrm{ie}} \approx 500 \mathrm{meV}$ and a critical current density of the SINS stack of $j_{\mathrm{c}}=200 \mathrm{~A} \mathrm{~cm}^{-2}$. This indicates a decrease in $I_{\mathrm{c}} R_{\mathrm{n}}$ by a factor of 2 caused by the F-layer interface scattering compared with SIS or SINFS JJs. In the case of SIFS JJs with the magnetically weaker $\mathrm{Ni}$ as interlayer, a decrease by a factor of $\sim 5$ was determined [17]. 
In the thickness range $d_{\mathrm{F}}>d_{0}=0.6 \mathrm{~nm}$ the ferromagnetism sets in (see figure 4) and the theory for SIFS junctions applies. From the envelope of the function $I_{\mathrm{c}}\left(d_{\mathrm{F}}\right)$ following equation (1), which was applied to our data points in the thickness range between $d_{\mathrm{F}}=0.6-1.7 \mathrm{~nm}$, we can estimate the damping length $\xi_{\mathrm{F} 1} \approx 0.22 \mathrm{~nm}$ (see figure 5). Obviously, the damping in the magnetic part of the film is stronger than in the non-magnetic part $\left(\xi_{\mathrm{N}}=0.28 \mathrm{~nm}\right)$. This indicates the effect of the strong exchange interaction on the pair density.

For a quantitative comparison with theory the model for SIFS junctions can be adapted to our system by considering the IN ayers between $\mathrm{Nb}$ and $\mathrm{Fe}-\mathrm{Co}$ as one interface with a very low transparency. In this model the oscillation length $\xi_{\mathrm{F} 2}=0.79 \mathrm{~nm}$ is definitely larger than the decay length $\xi_{\mathrm{F} 1}=$ $0.22 \mathrm{~nm}$, indicating a considerable influence of pair breaking scattering on the tunnelling. Using the experimental value for $\xi_{\mathrm{F} 1}, \xi_{\mathrm{F} 2}$ and $\ell_{\mathrm{m}}=0.23 \mathrm{~nm}$ we calculate $E_{\mathrm{ex}}=453 \mathrm{meV}$ and $\hbar / \tau_{\text {ie }}=750 \mathrm{meV}$. The ratio $\left(\hbar / \tau_{\text {ie }}\right) / E_{\text {ex }}=1.66$ is slightly larger but has the same magnitude as the corresponding value of $\mathrm{NiCu}$ where $\hbar / \tau_{\mathrm{ie}} \approx 1.33 \cdot E_{\mathrm{ex}}^{\mathrm{NiCu}} \approx 100 \mathrm{meV}$ has been determined [14]. Obviously the scattering energy of the $\mathrm{NiCu}$ alloy is 7.5 times smaller than the one for $\mathrm{Fe}-\mathrm{Co}$. The value obtained for the exchange energy $E_{\mathrm{ex}}=453 \mathrm{meV}$ is found to be significantly larger than for the elemental magnets $\mathrm{Ni}$ (80-200 meV), Co (309 meV) or Fe (256 meV) [16]. This seems reasonable considering the strong magnetic properties of $\mathrm{Fe}_{0.75} \mathrm{Co}_{0.25}$.

It should be mentioned that although the 0 to $\pi$ transition is located below the instrumental resolution limit of $0.1 \mu \mathrm{V}$, the thickness dependence in figure 5 suggests a crossover $0-\pi$ at $d_{\mathrm{F}} \approx 1.9 \mathrm{~nm}$. The experimental curve cannot be fitted reasonably by a pure exponential decay, a fit with equation (1) is much better. The calculated oscillation period $\Delta d_{\mathrm{F}}=2.48 \mathrm{~nm}$ fits perfectly into the series of values obtained for other systems such as $\Delta d_{\mathrm{F}}=3.7 \mathrm{~nm}$ [17] for pure $\mathrm{Ni}$ and $\Delta d_{\mathrm{F}}=11.0 \mathrm{~nm}$ [14] for the $\mathrm{Ni}_{0.53} \mathrm{Cu}_{0.47}$ alloy.

\section{Summary and conclusions}

We have shown that high quality JJs with the strong ferromagnetic alloy $\mathrm{Fe}-\mathrm{Co}$ as the barrier layer can be grown. The high quality of the barriers has been demonstrated by the observation of excellent Fraunhofer patterns in the case of nonmagnetic or magnetically weak, i.e. very thin F layers. The JJs are characterized by a strong damping of the superconducting pair amplitude in the $\mathrm{Fe}-\mathrm{Co}$ layers and a small oscillation period for the transition between 0 coupling and $\pi$ coupling. The strong damping of the pair amplitude even for the thickness range below $d_{\mathrm{F}}=0.6 \mathrm{~nm}$, where the $\mathrm{Fe}-$ Co layers appear to be non-ferromagnetic in the hysteresis loops, is probably caused by pair breaking scattering on magnetic fluctuations, which exist in itinerant ferromagnets close to a ferromagnetic phase boundary. The ferromagnetic range, too, is characterized by strong inelastic pair breaking scattering of the Cooper pairs, indicating a high density of states for low energy magnetic excitations in the $\mathrm{Fe}-\mathrm{Co}$ ferromagnetic layer with a thickness of only a few monolayers. Additionally, we observe non-reproducible flux trapping and intrinsic magnetization effects.

Applying the dirty limit model to our data gives a reasonable set of parameters and a consistent picture of our system. Although the 0 to $\pi$ transition of the $\mathrm{JJ}$ was not directly observable due to the strong damping of the critical current density $j_{\mathrm{c}}$ we could estimate its position at $d_{\mathrm{F}} \approx 1.9 \mathrm{~nm}$.

\section{Acknowledgments}

The authors thank R Waser, G Pickartz and R Borowski for support. DS, KW and HZ acknowledge financial support by SFB-491, and MW by project WE 4359/1-1.

\section{References}

[1] Buzdin A I 2005 Rev. Mod. Phys. 77935

[2] Tagirov L R 1998 Physica C 307145

[3] Zdravkov V, Sidorenko A, Obermeier G, Gsell S, Schreck M, Müller C, Horn S, Tidecks R and Tagirov L R 2006 Phys. Rev. Lett. 97057004

[4] Westerholt K, Sprungmann D, Zabel H, Brucas R, Hjörvarsson B, Tikhonov D A and Garifullin I A 2005 Phys. Rev. Lett. 95097003

[5] Weides M, Kemmler M, Goldobin E, Koelle D, Kleiner R, Kohlstedt H, and Buzdin A 2006 Appl. Phys. Lett. 89122511

[6] Vasenko A S, Golubov A A, Kupriyanov M Y and Weides M 2008 Phys. Rev. B 77134507

[7] Ryazanov V V, Oboznov V A, Rusanov A Y, Veretennikov A V, Golubov A A and Aarts J 2001 Phys. Rev. Lett. 862427

[8] Sellier H, Baraduc C, Lefloch F and Calemczuk R 2003 Phys. Rev. B 68054531

[9] Blum Y, Tsukernik A, Karpovski M and Palevski A 2002 Phys. Rev. Lett. 89187004

[10] Kontos T, Aprili M, Lesueur J, Genet F, Stephanidis B and Boursier R 2002 Phys. Rev. Lett. 89137007

[11] Ustinov A V and Kaplunenko V K 2003 J. Appl. Phys. 945405

[12] Yamashita T, Tanikawa K, Takahashi S and Maekawa S 2005 Phys. Rev. Lett. 95097001

[13] Weides M, Kemmler M, Goldobin E, Kohlstedt H, Waser R, Koelle D and Kleiner R 2006 Phys. Rev. Lett. 97247001

[14] Oboznov V A, Bol'ginov V V, Feofanov A K, Ryazanov V V and Buzdin A I 2006 Phys. Rev. Lett. 96197003

[15] Vávra O, Gaži S, Golubović D S, Vávra I, Dérer J, Verbeeck J, Tendeloo G V and Moshchalkov V V 2006 Phys. Rev. B 74020502

[16] Robinson J W A, Piano S, Burnell G, Bell C and Blamire M G 2007 Phys. Rev. B 7694522

[17] Bannykh A A, Pfeiffer J, Stolyarov V S, Batov I E, Ryazanov V V and Weides M 2008 arXiv:0808.3332

[18] Weides M, Tillmann K and Kohlstedt H 2006 Physica C 437-438 349

[19] Kroger H, Smith L N and Jillie D W 1981 Appl. Phys. Lett. 39280

[20] Pippard A B 1960 Rep. Prog. Phys. 23176

[21] Kim K, Kwon J H, Kim J, Char K, Doh H and Choi H-Y 2006 Phys. Rev. B 74174503

[22] Kim J, Kwon J H, Char K, Doh H and Choi H Y 2005 Phys. Rev. B 7214518

[23] Weides M 2008 Appl. Phys. Lett. 9352502 